\title{
Orexin Neurons Function in an Efferent Pathway of a Food- Entrainable Circadian Oscillator in Eliciting Food- Anticipatory Activity and Wakefulness
}

\author{
Michihiro Mieda, ${ }^{1,4}$ S. Clay Williams, ${ }^{1,4}$ Christopher M. Sinton, ${ }^{2}$ James A. Richardson, ${ }^{3}$ Takeshi Sakurai,, 5 and \\ Masashi Yanagisawa ${ }^{1,4,6}$ \\ Departments of ${ }^{1}$ Molecular Genetics, ${ }^{2}$ Internal Medicine, and ${ }^{3}$ Pathology, and ${ }^{4}$ Howard Hughes Medical Institute, University of Texas Southwestern \\ Medical Center, Dallas, Texas 75390-9050, ${ }^{5}$ Department of Pharmacology, Institute of Basic Medical Sciences, University of Tsukuba, Ibaraki 305-8575, \\ Japan, and ${ }^{6}$ Exploratory Research for Advanced Technology Yanagisawa Orphan Receptor Project, Japan Science and Technology Agency, Tokyo 135-0064, \\ Japan
}

Temporal restriction of feeding can entrain circadian behavioral and physiological rhythms in mammals. Considering the critical functions of the hypothalamic orexin (hypocretin) neuropeptides in promoting wakefulness and locomotor activity, we examined the role of orexin neurons in the adaptation to restricted feeding. In orexin neuron-ablated transgenic mice, the food-entrained rhythmicity of $m$ Per 2 expression in the brain and liver, the reversal of the sleep-wake cycle, and the recovery of daily food intake were unaltered compared with wild-type littermates. In contrast, orexin neuron-ablated mice had a severe deficit in displaying the normal foodanticipatory increases in wakefulness and locomotor activity under restricted feeding. Moreover, activity of orexin neurons markedly increased during the food-anticipatory period under restricted feeding in wild-type mice. Orexin neurons thus convey an efferent signal from putative food-entrainable oscillator or oscillators to increase wakefulness and locomotor activity.

Key words: circadian rhythm; sleep-wake; orexin/hypocretin; food anticipatory activity; food-entrainable oscillator; restricted feeding

\section{Introduction}

The circadian oscillator in the suprachiasmatic nucleus (SCN), which is regarded as the master clock in mammals, orchestrates multiple circadian biological rhythms in the organism and is regulated according to environmental light/dark cues conveyed from the eye [light-entrainable oscillator (LEO)] (Buijs and Kalsbeek, 2001; Reppert and Weaver, 2002). However, when food availability is restricted to a single period scheduled at a fixed time of the day (restricted feeding), animals adapt to this condition within a few days by feeding during the period of food availability and by increasing locomotor activity in the preceding hours [food-anticipatory activity (FAA)] (Mistlberger, 1994; Stephan, 2002). Such anticipatory behaviors have been documented in multiple mammalian and avian species and are often accompanied by increases in body temperature, adrenal corticosterone

Received Aug. 3, 2004; revised Sept. 14, 2004; accepted Sept. 22, 2004.

This work was supported in part by research funds from Keck Foundation, the Perot Family Foundation, and the Exploratory Research for Advanced Technology/Japan Science and Technology Agency. M.Y. is an Investigator of the Howard Hughes Medical Institute. M.M. is a long-term fellow of the Human Frontier Science Program. We thank S. L. McKight, M. S. Brown, J. L. Goldstein, and J. T. Willie for valuable discussions and S. A. Dixon, R. O. Floyd, and M. Thornton for technical support.

M. Mieda's present address: Department of Molecular Neuroscience, Medical Research Institute, Tokyo Medical and Dental University, 1-5-45 Yushima, Bunkyo-ku, Tokyo 113-8519, Japan.

Correspondence should be addressed to Masashi Yanagisawa, Howard Hughes Medical Institute, University of Texas Southwestern Medical Center, 5323 Harry Hines Boulevard, Dallas, TX 75390-9050. E-mail: Masashi.Yanagisawa@UTSouthwestern.edu.

D0I:10.1523/JNEUROSCI.3171-04.2004

Copyright $(\odot 2004$ Society for Neuroscience $\quad 0270-6474 / 04 / 2410493-09 \$ 15.00 / 0$ secretion, gastrointestinal motility, and activity of digestive enzymes. These changes in biological rhythms have been postulated to be brought about by a food-entrainable oscillator (FEO) that is independent of the SCN, although little is known of the physical substrates of the FEO. Recent studies demonstrated that restricted feeding during the light phase entrains and shifts the circadian rhythms of gene expression in the peripheral tissues as well as in certain brain regions other than the SCN, whereas rhythmicity in the SCN remained phase locked to the light/dark cycle. This suggests a phase uncoupling of multiple circadian oscillators outside the SCN from the master clock under restricted feeding (Damiola et al., 2000; Stokkan et al., 2001; Wakamatsu et al., 2001).

Little is known regarding the efferent pathways through which the putative FEO may modulate behavior. Orexin-A and - B (hypocretin-1 and -2) are neuropeptides produced by cleavage of a single precursor prepro-orexin, and they act on two orexin receptors, orexin receptor type $1\left(\mathrm{OX}_{1} \mathrm{R}\right)$ and type $2\left(\mathrm{OX}_{2} \mathrm{R}\right)(\mathrm{de}$ Lecea et al., 1998; Sakurai et al., 1998). Intracerebroventricular administration of these peptides in rodents elevates locomotor activity and wakefulness (Hagan et al., 1999). Neurons producing orexins are located exclusively in the lateral and posterior hypothalamus and send their projections widely throughout the CNS, including a number of nuclei involved in vigilance state regulation (Peyron et al., 1998; Chemelli et al., 1999; Date et al., 1999). The importance of the orexin system in promoting wakefulness is highlighted by the findings that mice lacking either the orexin gene (Chemelli et al., 1999) or orexin neurons (Hara et al., 2001), 
as well as mice and dogs with null mutations in the $\mathrm{OX}_{2} \mathrm{R}$ gene (Lin et al., 1999; Willie et al., 2003), show phenotypes remarkably similar to the human sleep disorder narcolepsy (Mignot et al., 2002; Scammell, 2003).

We previously described a transgenic mouse strain (designated here as orexinergic(-) mice) in which the hypothalamic orexin neurons are specifically and postnatally ablated through the expression of a poly-Gln-containing fragment of the ataxin-3 polypeptide, the product of Machado-Joseph disease gene (Hara et al., 2001). In the present study, we have examined the role of orexin neurons in the adaptation to restricted feeding using orexinergic(-) mice (Mistlberger et al., 2003).

\section{Materials and Methods}

Animals. Orexinergic(-) mice were generated as described previously (Hara et al., 2001). Mice used for all experiments in this study were 13- to 20 -week-old male orexinergic(-) hemizygous mice and their wild-type littermates (C57BL/6J × 129SvEv F1 genetic background), generated by crosses between male orexinergic(-) hemizygous mice (N4-N7 backcross to C57BL/6J) and wild-type female mice (129SvEv). All animal procedures were approved by the appropriate institutional animal care and use committees and were performed in strict accordance with National Institutes of Health guidelines.

Protocols for restricted feeding. Mice were kept on a $12 \mathrm{hr}$ light/dark cycle. Before recording, all mice were individually housed for at least $7 \mathrm{~d}$ and then monitored for electroencephalograph-electromyograph (EEG-EMG) activity or locomotor activity from the onset of the dark phase [zeitgeber time (ZT) 12; ZT0 is defined as the lights-on time and ZT12 as the lights-off time] for $48 \mathrm{hr}$ under ad libitum feeding (ALF) (days -2 and -1$)$. During ALF recording, two food pellets $(\sim 5.5 \mathrm{gm}$ each) were put on the cage floor and replaced with fresh ones every day at ZT4 (in light phase-restricted feeding experiments; LRF) or ZT16 (in dark phase-restricted feeding experiments; DRF). Pellets were also handled very briefly at ZT8 (LRF experiments) or ZT20 (DRF experiments) to mimic the procedures under restricted feeding. On the next day (day 0 ), food pellets were replaced with fresh ones as on days -2 and -1 and then removed at ZT8 (LRF) or ZT20 (DRF). From day 1, two food pellets were put on the cage floor at ZT4 (LRF) or ZT16 (DRF) and then removed at ZT8 (LRF) or ZT20 (DRF). Data for restricted feeding were collected on days 8 and 9 . After restricted feeding recording, data were collected for a further $24 \mathrm{hr}$ (LRF) or $12 \mathrm{hr}$ (DRF) without food (RF-fast), followed by refeeding. During this part of the study, the experimenter again put his hand in the cages at ZT4 and ZT8 (LRF) or ZT16 and ZT20 (DRF) to mimic the procedures under restricted feeding. Because preliminary EEG-EMG recordings indicated that sleep-wake rhythms after 1 and 2 weeks of LRF were essentially the same, we collected data after 1 week of restricted feeding.

Gene expression analyses. Neither locomotor activity nor EEG-EMG was recorded from mice used for tissue collection. Tissues were collected in duplicate (two mice per data point) from wild-type and orexinergic(-) littermates under ALF or LRF at ZT1, 7, 13, and 19 (for the brain) or at ZT7 and 15 (for the liver) on day 9 (see protocols for restricted feeding). For tissue collection from mice under ALF, mice continued to be fed ad libitum from day 0 to day 9. In situ hybridization was performed as described (Chemelli et al., 1999). A $0.3 \mathrm{~kb}$ fragment of mPer 2 cDNA was used to generate sense (control) and antisense riboprobes (Reick et al., 2001). For the quantitative reverse transcription (RT)-PCR, total RNA was prepared from mouse livers using the RNA STAT-60 kit (Tel-Test, Friendswood, TX). cDNA was synthesized from $2 \mu \mathrm{g}$ of total RNA using oligo $(\mathrm{dT})_{12-18}$ primers and the SuperScript II reverse transcriptase (Invitrogen, Carlsbad, CA). mPer2 and mouse glyceraldehydes-3-phosphate dehydrogenase $(G A P D H)$ cDNA fragments were amplified and quantified by real-time PCR from 1/50 of synthesized cDNA mixtures using the ABI PRISM 7000 Sequence Detection System and the SYBR Green PCR Master Mix (Applied Biosystems, Foster City, CA). The primer sequences were: $m$ Per2 upstream, 5'-TCCTGTCATGGCCTTCATGCT-3'; mPer2 downstream, 5'-GCAGGAGTTATTTCGGAGGCA-3'; GAPDH upstream, 5'-GTGGCAAAGTGGAGATTGTTGCC-3'; GAPDH downstream, 5' -GATGATGACCCGTTTGGCTCC-3'.

Measurement of locomotor activity. Open-field locomotor activity was recorded, and then the distances traveled were calculated using the AccuScan (Columbus, OH) VersaMax system. Mice were transferred from home cages into the apparatus for habituation $4 \mathrm{hr}$ before the recording period under ALF that started at the onset of the dark phase and kept in the apparatus throughout the experiments (see "Protocols for restricted feeding").

$E E G-E M G$ recordings. Mice were anesthetized and surgically implanted for long-term EEG-EMG monitoring as described previously (Chemelli et al., 1999). EEG-EMG signals were amplified, filtered, digitized, archived, and scored into standard vigilance states as described and further analyzed using custom software (Chemelli et al., 1999). For calculation of the hourly mean durations of wakefulness episodes, we treated each wakefulness episode that crossed the boundary between the hourly windows as two separate episodes.

Fos mapping. Neither locomotor activity nor EEG-EMG was recorded from mice used for tissue collection. Brains were collected (five mice per each group) from wild-type and orexinergic(-) littermates under ALF or LRF at ZT3.5 on days 8, 9, and 10 (see Protocols for restricted feeding). For tissue collection from mice under ALF, mice continued to be fed ad libitum from day 0 to day 10. Double staining for orexin and Fos was performed as described (Beuckmann et al., 2004), using anti-orexin (Chemelli et al., 1999) and anti-Fos (Ab-5; 1:50,000 dilution) (Oncogene Research Products, Cambridge, MA) antibodies. Numbers of stained cells in the perifornical area, LC (bilateral counting from one section per sample), and the tuberomammillary nucleus (TMN) (bilateral counting from three sections $90 \mu \mathrm{m}$ apart per sample) were counted using a Bioquant (Nashville, TN) Nova system.

Statistical analysis. Activity and vigilance state data were initially analyzed by two-way repeated-measures ANOVA to detect effects of genotype, feeding conditions, and their interactions. For significant measures, initial analysis was followed by one-way repeated-measures ANOVA and Tukey post hoc tests to detect effects of feeding conditions within each genotype and by two-way repeated-measures ANOVA to detect interactions between genotype and each combination of two of the multiple feeding conditions. The criterion for rejection of the null hypothesis was $p<0.05$.

\section{Results}

\section{Orexin neurons are not required for circadian entrainment by feeding}

To detect any differences between wild-type and orexinergic(-) mice (Hara et al., 2001) in the entrainment of circadian oscillators by restricted feeding, we first compared circadian rhythms of $m$ Per 2 expression in the brain and liver of wild-type and orexinergic(-) mice under ALF or under $4 \mathrm{hr}$ restricted feeding in the middle of the light phase (ZT4-8: LRF; see Materials and Methods). We collected brains at four time points (ZT1, 7, 13, and 19) on the ninth day under ALF or LRF and performed in situ hybridization. These four sampling time points were sufficient to detect the overt qualitative differences in temporal expression patterns between different feeding conditions. In the SCN of wild-type mice, we observed weak expression of mPer2 at ZT1, highest levels at ZT7, moderate levels at ZT13, and essentially no expression at ZT19 under ALF. As previously reported, LRF did not appreciably change the mPer2 rhythm in the SCN (Fig. 1a). In contrast, $m$ Per 2 expression in the striatum and cerebral cortex was higher in the dark phase $(\mathrm{ZT} 13,19)$ under ALF, whereas under LRF expression was highest at ZT7 (during food availability), with expression levels apparently higher than those at peak under ALF. These temporal patterns of mPer 2 expression are consistent with previous reports and indicate a phase uncoupling of circadian oscillators outside the SCN from the SCN clock under LRF (Zylka et al., 1998; Damiola et al., 2000; Reick et al., 2001; 


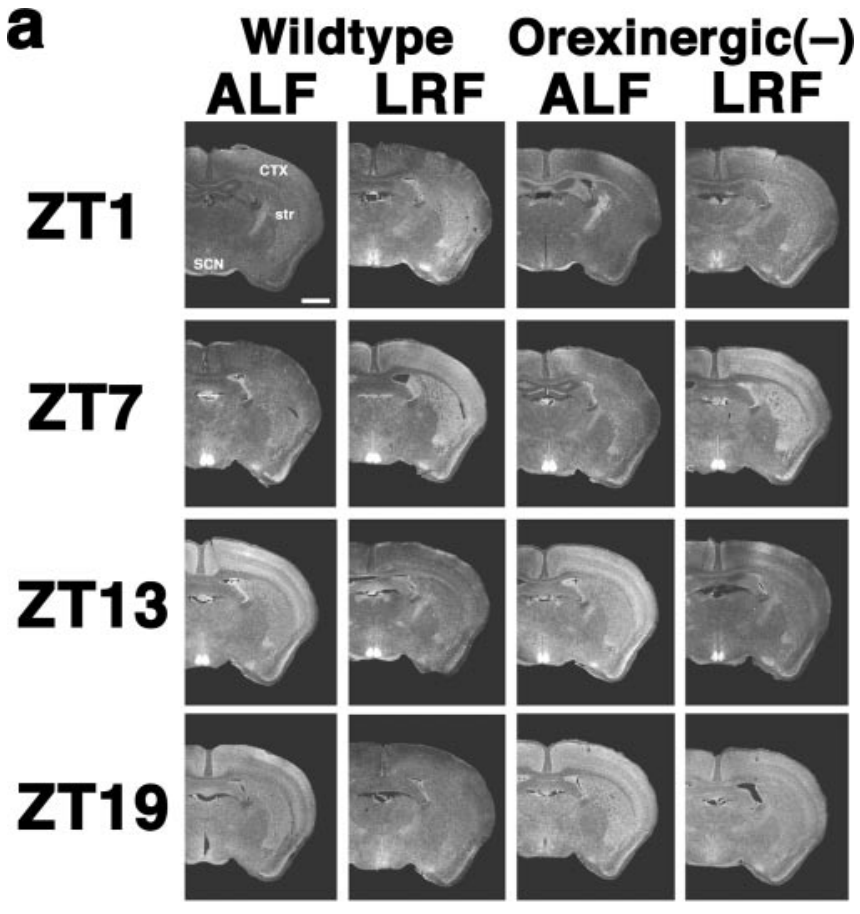

b

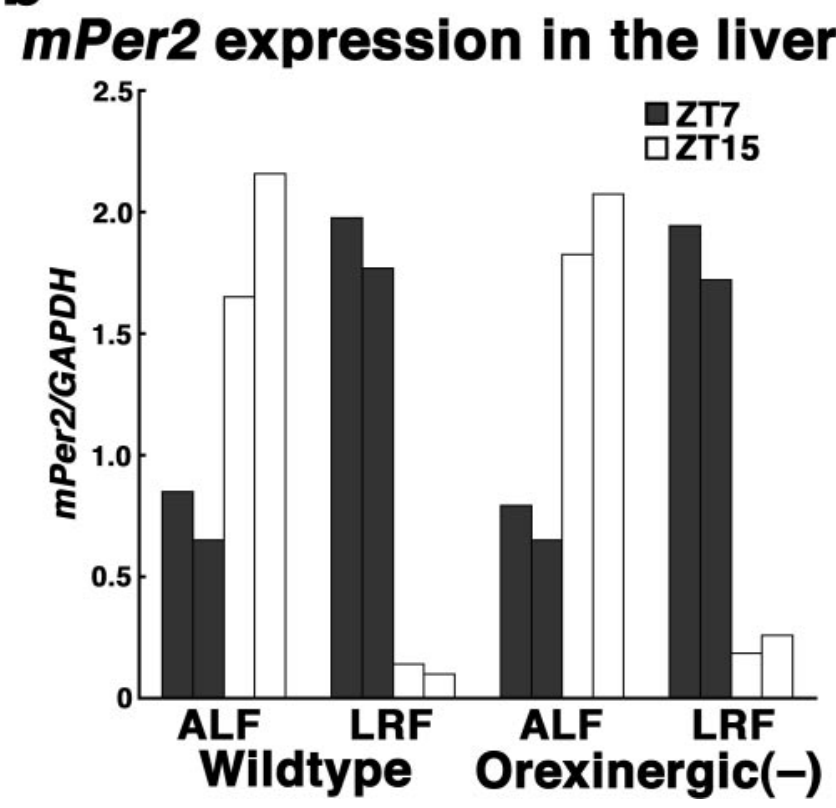

Figure 1. Normal food entrainment of central and peripheral $m$ Per2 expression in orexinergic(-) mice. $a$, Coronal brain sections at the level of the $S C N$, prepared from wild-type and orexinergic(-) mice under ALF or LRF at ZT1, 7, 13, and 19, were hybridized in situ to a ${ }^{35} \mathrm{~S}$ labeled mPer2 antisense probe. CTX, Cerebral cortex; str, striatum. Scale bar, 1 mm. b, mPer2 mRNA in the livers of wild-type and orexinergic(-) mice under ALF or LRF at ZT7 and 15 was quantified by real-time RT-PCR. Values from two mice per data point are shown. Each value was normalized to GAPDH mRNA.

Stokkan et al., 2001; Wakamatsu et al., 2001). In the brains of orexinergic(-) mice, the temporal patterns of mPer2 expression were essentially identical to those of wild-type mice as described above.

We then quantified mPer2 mRNA in the livers collected at ZT7 and ZT15 by real-time RT-PCR (Fig. 1b). Under ALF, mPer2 expression was much higher at ZT15 than ZT7 in both genotypes.


Figure 2. Normal changes to vigilance states and food intake in orexinergic(-) mice under light phase-restricted feeding. $a$, Time spent in each vigilance state during $24 \mathrm{hr}$ day (24-h), 12 hr dark phase (Dark), and $12 \mathrm{hr}$ light phase (Light). Note that under LRF, the light/dark rhythm of the sleep-wake cycle is reversed compared with that under ALF in both wild-type (wt) and orexinergic(-) (ox-) mice. In addition, orexinergic(-) mice show reduced total $24 \mathrm{hr}$ wakefulness time. ${ }^{* * *} p<0.001$ ALF versus LRF within each genotype; ${ }^{\# \#} p<0.01 ;{ }^{\# \# \#} p<0.001$ interaction of feeding condition and genotype; two-way repeated-measures ANOVA. Values are means $\pm S E$ ( $n=8$ for each genotype). $b$, Amount of food consumed by wild-type or orexinergic(-) mice relative to those on day -1 . Values at each time point are not significantly different between genotypes by unpaired $t$ test. From days -3 to -1 , under ALF; on day 0 , food was removed at ZT8; from day 1 to day 9 , under LRF. Values are means \pm SE ( $n=8$ for each genotype) that were monitored during the EEG-EMG recording experiments (Figs. 2a, 4). Food intake during locomotor activity measurements (Fig. 3) exhibited curves very similar to those shown here.

The light/dark rhythm of mPer2 expression in the liver was completely reversed under LRF in both genotypes, as reported previously for normal mice and rats (Damiola et al., 2000; Stokkan et al., 2001). Collectively, these results suggest that LRF entrained the molecular clocks similarly in wild-type and orexinergic(-) mice.

We next monitored vigilance state patterns of wild-type and orexinergic(-) mice by recording EEG-EMG activity under ALF and LRF. The $24 \mathrm{hr}$ wakefulness time in wild-type mice was similar in LRF and ALF (Fig. 2a). In contrast, LRF caused a significant reduction in total $24 \mathrm{hr}$ wakefulness time compared with that under ALF in orexinergic(-) mice; this reduction in $24 \mathrm{hr}$ wakefulness time under LRF was accompanied by an increase in $24 \mathrm{hr}$ non-REM sleep time but not in $24 \mathrm{hr}$ REM sleep time. The time spent in wakefulness under ALF was not significantly different between the two genotypes, as described previously (Hara et al., 2001). Wakefulness time during the $12 \mathrm{hr}$ dark/light phases showed that, as expected, both wild-type and orexinergic(-) mice were more awake during the dark (i.e., active) phase than during the light (i.e., rest) phase under ALF. Importantly, under LRF, sleep-wake rhythms were reversed in both genotypes (Fig. 2a); reduction of wakefulness in the dark phase under LRF was com- 

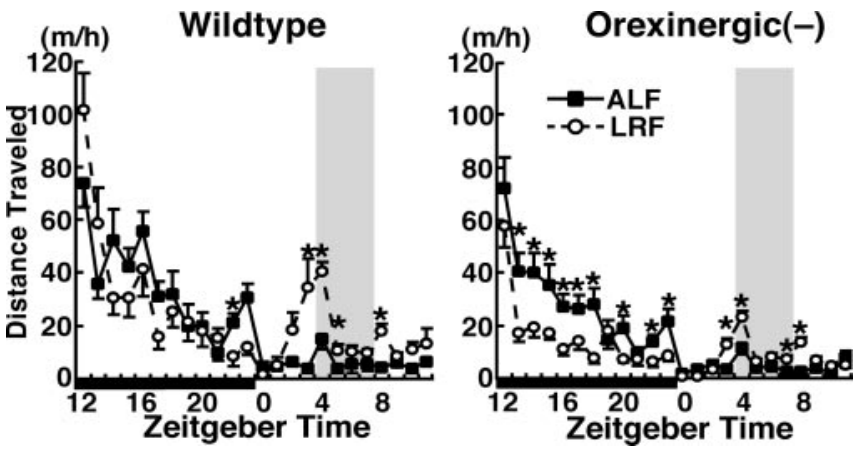

Figure 3. Attenuation of food-anticipatory locomotor activity in orexinergic(-) mice under light phase-restricted feeding. Hourly plots of distance traveled by wild-type and orexinergic(-) mice under ALF or LRF. The period of food availability is shaded. The dark phase is indicated by a solid horizontal bar. ${ }^{*} p<0.05$ ALF versus LRF within each genotype; one-way repeatedmeasures ANOVA. Values are means $\pm S E(n=12$ for each genotype). By two-way repeatedmeasures ANOVA, the interaction of condition and genotype at ZT2 is significant ( $p=0.0429$ ) but did not reach significance at ZT3 ( $p=0.0502$ ).

parable between the two genotypes, accompanied by similar increases in non-REM sleep time in the dark phase. However, the increase in wakefulness in the light phase under LRF was less in the orexinergic(-) mice than in the wild-type mice because of attenuation of the food-anticipatory increase in wakefulness (see below). Nevertheless, these data suggested that food-entrainment of circadian sleep-wake rhythms was not fundamentally impaired in orexinergic $(-)$ mice.

Under LRF, wild-type and orexinergic(-) mice showed similar changes in daily food intake and body weight. During the first day of LRF, they ate only $\sim 10 \%$ of the food that they ate under ALF (Fig. 2b). However, mice of both genotypes rapidly increased food intake to reach $\sim 85 \%$ of ALF intake after $9 \mathrm{~d}$ of LRF. The absolute food intake was significantly smaller in orexinergic(-) mice than in wild-type mice under LRF as well as under ALF, as reported previously (Hara et al., 2001) (5.3 \pm 0.2 vs $4.5 \pm 0.1 \mathrm{gm}$ under ALF, $p=0.002, n=8 ; 4.5 \pm 0.2$ and $3.8 \pm 0.1$ gm after $9 \mathrm{~d}$ under LRF, $p=0.007, n=8$; unpaired $t$ test). Body weights were not significantly different between the genotypes (30.8 \pm 0.5 and $30.7 \pm 1.6 \mathrm{gm}$ before LRF; $26.4 \pm 0.5$ and $26.7 \pm 1.5 \mathrm{gm}$ after $5 \mathrm{~d}$ under LRF). Thus, metabolic aspects of adaptation to LRF seemed comparable between wild-type and orexinergic(-) mice. These changes under LRF in food intake, body weight, central and peripheral $m P e r 2$ expression, and circadian rhythms of the sleep-wake cycle all suggested that orexin neurons are not required for circadian entrainment by restricted feeding.

Orexin neurons are essential for food-anticipatory circadian behaviors

We next examined the hourly distribution of locomotor activity and wakefulness time in wild-type and orexinergic(-) mice under ALF or LRF, to examine the role of orexin neurons in the foodanticipatory increase in activity and wakefulness. Under LRF, wild-type mice demonstrated robustly increased locomotor activity for $2 \mathrm{hr}$ preceding food availability (Fig. 3 ) and increased the time spent in wakefulness for $3 \mathrm{hr}$ preceding food availability (Fig. 4a,b). In contrast, orexinergic(-) mice exhibited severely blunted anticipatory increases in both locomotor activity and wakefulness time that were restricted to the $1 \mathrm{hr}$ period preceding food availability (Figs. 3, 4a,b). These data suggested that orexin neurons are essential for the normal food-anticipatory increase in activity and wakefulness under LRF. We focused on vigilance state regulation for additional study, because, under our experi-
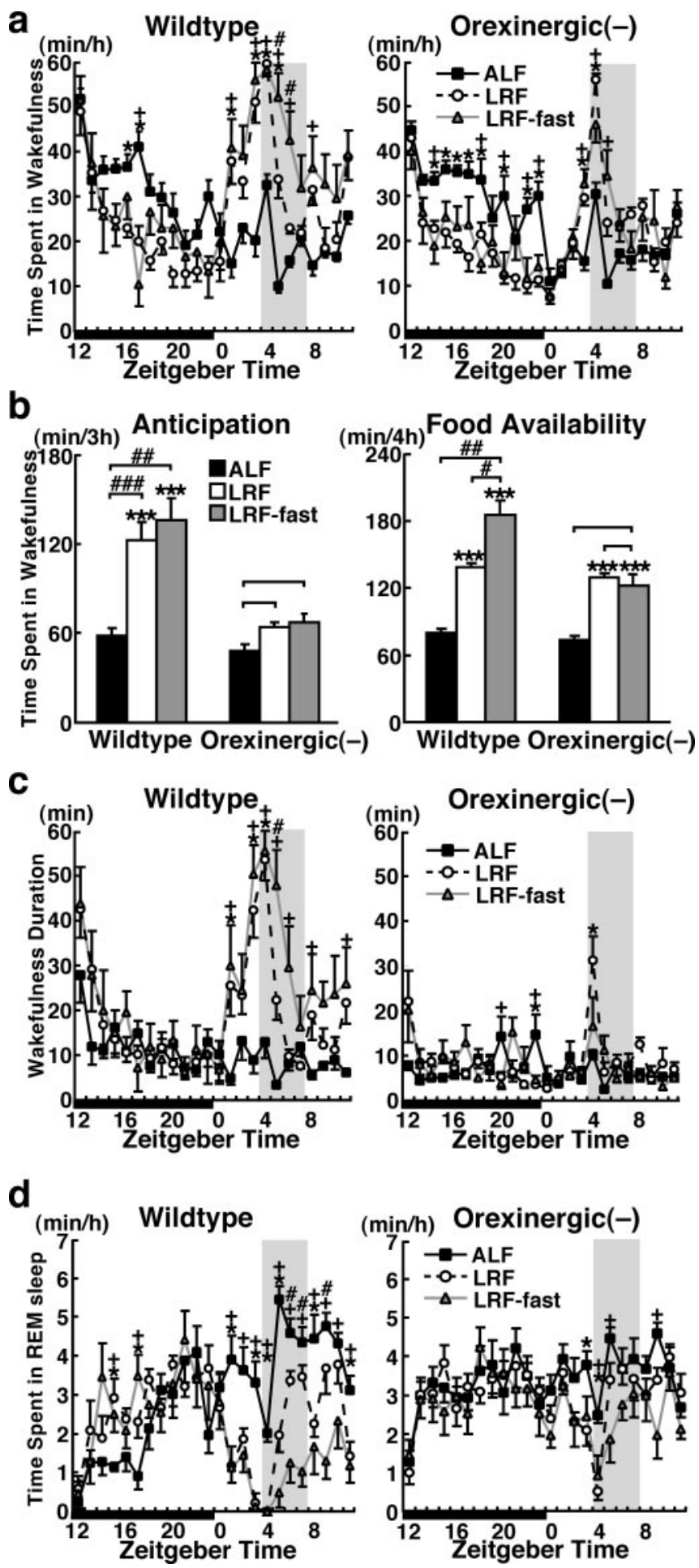

Figure 4. Attenuation of food-anticipatory increase in wakefulness in orexinergic(-) mice under light phase-restricted feeding. $a$, Hourly plots of time spent in wakefulness under ALF, LRF, or LRF without food (LRF-fast; see Results and Materials and Methods). $b$, Time spent in wakefulness during the periods of anticipation (ZT1-4) or food availability (ZT4-8). c, Hourly plots of mean wakefulness episode duration. $d$, Hourly plots of time spent in REM sleep. In a, $c$, and $d$, the period of food availability is shaded, and the dark phase is indicated by a solid horizontal bar. ${ }^{*} p<0.05$ ALFversus LRF; ${ }^{+} p<0.05$ ALFversus LRF-fast; ${ }^{*} p<0.05$ LRF versus LRF-fast; within each genotype; one-way repeated-measures ANOVA and Tukey post hoc tests. $\ln b^{*}{ }^{* *} p<0.001$ compared with ALF within each genotype; ${ }^{\#} p<0.05 ;{ }^{\# \#} p<0.01$; ${ }^{\# \# \#} p<$ 0.001 interaction of condition and genotype; two-way repeated-measures ANOVA. Values are means \pm SE ( $n=8$ for each genotype). 
a

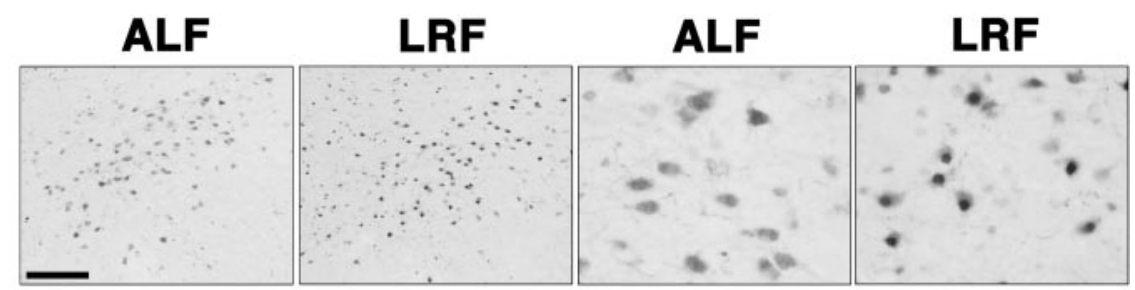

b

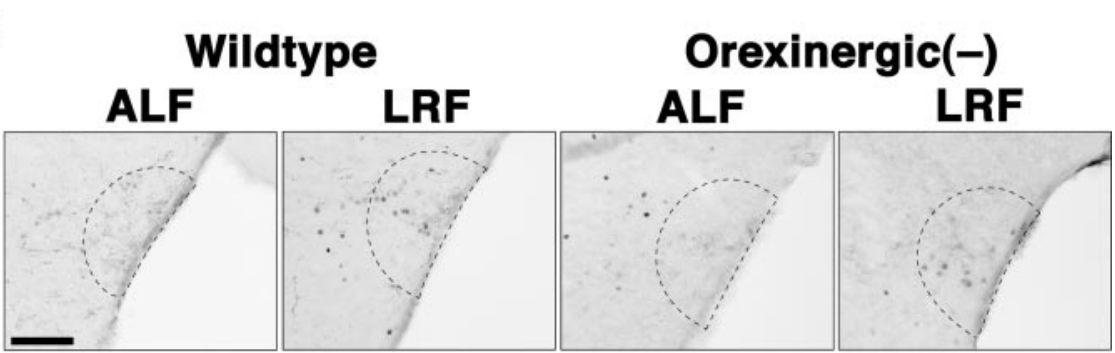

C
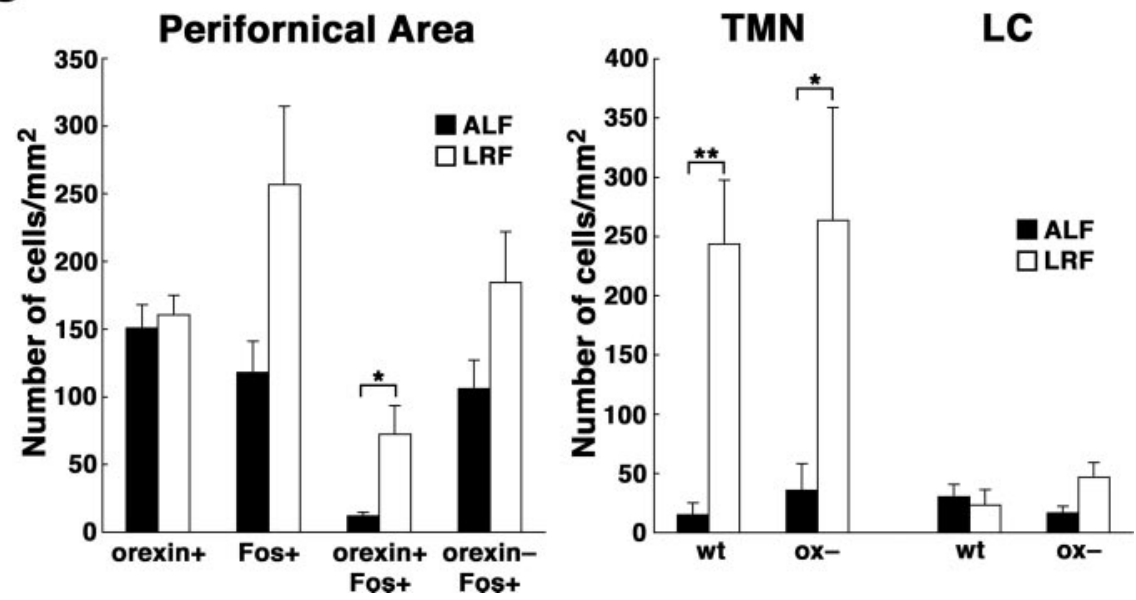

Figure 5. Activation of orexin neurons during the food-anticipatory period under light phase-restricted feeding. $a, 0$ rexin-IR cells (light staining in cytoplasm) and Fos-IR cells (dark staining in nuclei) in the perifornical area of wild-type mice at ZT3.5 under ALF or LRF. Areas shown in left two panels were defined by a rectangular box ventrolateral to the mammillothalamic tract, dorsal to the fornix, and medial to the optic nerve in the section at bregma $-1.46 \mathrm{~mm}$ (Franklin and Paxinos, 2001), and orexin-IR and/or Fos-IR cells in these areas were counted in c. Representative images with higher magnification are shown in the right two panels. $b$, Fos-IR cells in the TMN of wild-type and orexinergic(-) mice at ZT3.5 under ALF or LRF. Dense orexin-IR nerve terminals in the TMN (reticular staining) are seen in wild-type mice but not in orexinergic(-) mice. Areas defined as the TMN are shown by dotted lines. Scale bars: $a, 100 \mu \mathrm{m}$ for the left two images, $25 \mu \mathrm{m}$ for the right two images; $b, 50 \mu \mathrm{m}$. $c$, Numbers of orexin-IR and/or Fos-IR cells in the perifornical area in wild-type mice and Fos-IR cells in the TMN and LC of wild-type (wt) and orexinergic(-) (ox-) mice. ${ }^{*} p<0.05 ;{ }^{* *} p<0.01$ ALF versus LRF by unpaired $t$ test. Values are means \pm SE (5 mice for each group).

mental conditions in wild-type mice, the anticipatory increase in wakefulness time was more marked than that in locomotor activity.

Although orexinergic(-) mice exhibited a blunted foodanticipatory increase in wakefulness, they responded similarly to wild-type mice with respect to the time spent in wakefulness during the period of food availability (Fig. $4 a, b$ ). This suggested that orexin neurons are not required for the increased wakefulness while food is actually available. However, when mice were given no food during the expected feeding period after the establishment of FAA, wild-type mice significantly prolonged the period of increased wakefulness, but orexinergic(-) mice did not (Fig. $4 a, b$, LRF-fast).
Because orexin neurons have been implicated in the stabilization and maintenance of wakefulness episodes (Chemelli et al., 1999; Hara et al., 2001; Saper et al., 2001; Willie et al., 2003), we also plotted the mean hourly durations of these episodes (Fig. 4c). Wild-type mice showed markedly increased wakefulness episode durations for $3 \mathrm{hr}$ during the foodanticipatory period. In contrast, orexinergic (-) mice showed no increase in wakefulness episode durations until food was actually available. Thus, stabilization of wakefulness episodes may be the principal orexin-mediated mechanism for the foodanticipatory increase in wakefulness time. Hourly plots of the time spent in nonREM sleep were essentially the mirror images of those of wakefulness (data not shown). In wild-type mice, LRF significantly reduced non-REM sleep time for 3 hr preceding and during food availability, but non-REM sleep time was correspondingly increased in the dark phase. These changes resulted in a reversal of the light/ dark pattern for non-REM sleep time, although the total non-REM sleep time over $24 \mathrm{hr}$ remained unchanged (Fig. 2a). In orexinergic(-) mice, however, the reduction of non-REM sleep time during food anticipation was markedly blunted compared with wild-type mice, but the increase in this parameter in the dark phase was similar to that in wild-type mice. These changes resulted in an increase in 24 hr non-REM sleep time (Fig. 2a). Changes in REM sleep time resulting from LRF (Figs. $2 a, 4 d$ ) were approximately parallel to those noted for non-REM sleep time except that: (1) daily REM sleep time was reduced under LRF in both genotypes, and (2) orexinergic $(-)$ mice did not show increased REM sleep time in the dark phase under LRF despite the fact that non-REM sleep time was increased. As previously reported, orexinergic(-) mice spent more time in REM sleep than wild-type mice in the dark phase even under ALF (Fig. 2a) ( $p=0.0001$ by two-way ANOVA) (Hara et al., 2001). A ceiling effect might, therefore, be in operation, and further increases in REM sleep time in the dark phase under LRF would not be possible.

These results, suggesting an essential role of orexin neurons in displaying FAA, led us to examine whether orexin neurons are indeed activated during the food-anticipatory period by measuring Fos protein expression as an indicator of neuronal activity (Cirelli and Tononi, 2000). We collected brains of wild-type or orexinergic(-) mice at ZT3.5 under ALF or LRF (30 min before food availability in LRF) and sectioned and double-stained them with anti-Fos and anti-orexin antibodies. The number of orexinimmunoreactive (IR) cells in the perifornical area of wild-type mice was not different between ALF and LRF (Fig. 5a,c). Very few orexin-IR neurons, if any, were found in the same area of orex- 
inergic(-) mice, with much weaker staining (data not shown). In wild-type mice, the number of orexin-IR-Fos-IR double-labeled cells was increased 6.1-fold under LRF compared with that under ALF (Fig. 5a,c). The number of non-orexin-IR-Fos-IR cells in the same area was not significantly different under LRF in wild-type mice compared with that under $\operatorname{ALF}$ ( $p=0.11$ by unpaired $t$ test). These results indicated that orexin neurons are selectively activated among neurons within the perifornical area during the food-anticipatory period.

Because we observed a marked attenuation of foodanticipatory activity and wakefulness in orexinergic(-) mice, we also compared the number of Fos-IR cells between the two genotypes in some of the nuclei that have been implicated as direct downstream targets of orexin neurons in the regulation of the sleep-wake cycle. The number of Fos-IR cells in the TMN, which contains histaminergic neurons and is critical for the maintenance of wakefulness (Huang et al., 2001; Parmentier et al., 2002; Yamanaka et al., 2002), was dramatically increased during the food-anticipatory period under LRF compared with that under ALF in wild-type mice. Interestingly, orexinergic(-) mice exhibited similar degrees of LRF-induced increase in TMN Fos-IR (Fig. $5 b, c)$. This observation indicated that the activation of TMN neurons during food anticipation is independent of orexinergic projections. In contrast, only very few Fos-IR neurons were observed during the food-anticipatory period in the locus ceruleus (LC) (Fig. 5c) and the dorsal raphe nucleus (DR) (data not shown) of both wild-type and orexinergic(-) mice (Chemelli et al., 1999; Saper et al., 2001). These results in the TMN, LC, and DR of wild-type mice are consistent with a previous report in rats subjected to a similar scheme of food restriction (Inzunza et al., 2000).

Regulation of wakefulness by orexin neurons is modulated by circadian phase

During the initial dark phase under LRF (day 1; see Materials and Methods), which started $4 \mathrm{hr}$ after the first removal of food at ZT8, both wild-type and orexinergic(-) mice responded to the absence of food by increasing wakefulness (Fig. 6). Although the period of increased wakefulness lasted $1 \mathrm{hr}$ longer in wild-type mice than in orexinergic (-) mice (5 vs $4 \mathrm{hr}$ ), both genotypes exhibited a similar increase in wakefulness during the first $4 \mathrm{hr}$. Thus, although orexinergic(-) mice exhibited a failure to increase wakefulness during food anticipation when compared with wildtype mice under LRF (Fig. $4 a, b$ ), they, like wild-type mice, did appropriately increase wakefulness at the beginning of the first dark phase without food (Fig. 6a,b). An important difference between these two contexts is the time of the day when wakefulness needed to be increased. Interestingly, although the $24 \mathrm{hr}$ wakefulness time of orexinergic(-) mice was comparable to that of wild-type mice under ALF (a condition under which mice are more awake in the dark phase), total wakefulness time in orexinergic(-) mice was reduced under LRF, when the mice were more awake during the light phase (Fig. $2 a$ ).

To test the hypothesis that the orexin-mediated regulation of wakefulness is modulated by circadian phase, we examined the effect of a $4 \mathrm{hr}$ period of restricted feeding in the middle of the dark phase (ZT16-20, DRF) on the vigilance states of wild-type and orexinergic $(-)$ mice. The difference between genotypes in the food-anticipatory increase in wakefulness under DRF was similar to that under LRF. Thus, wild-type mice demonstrated increased wakefulness for $4 \mathrm{hr}$ preceding food availability under DRF, but orexinergic(-) mice exhibited a markedly blunted increase that was restricted to a $1 \mathrm{hr}$ period immediately preceding food avail-
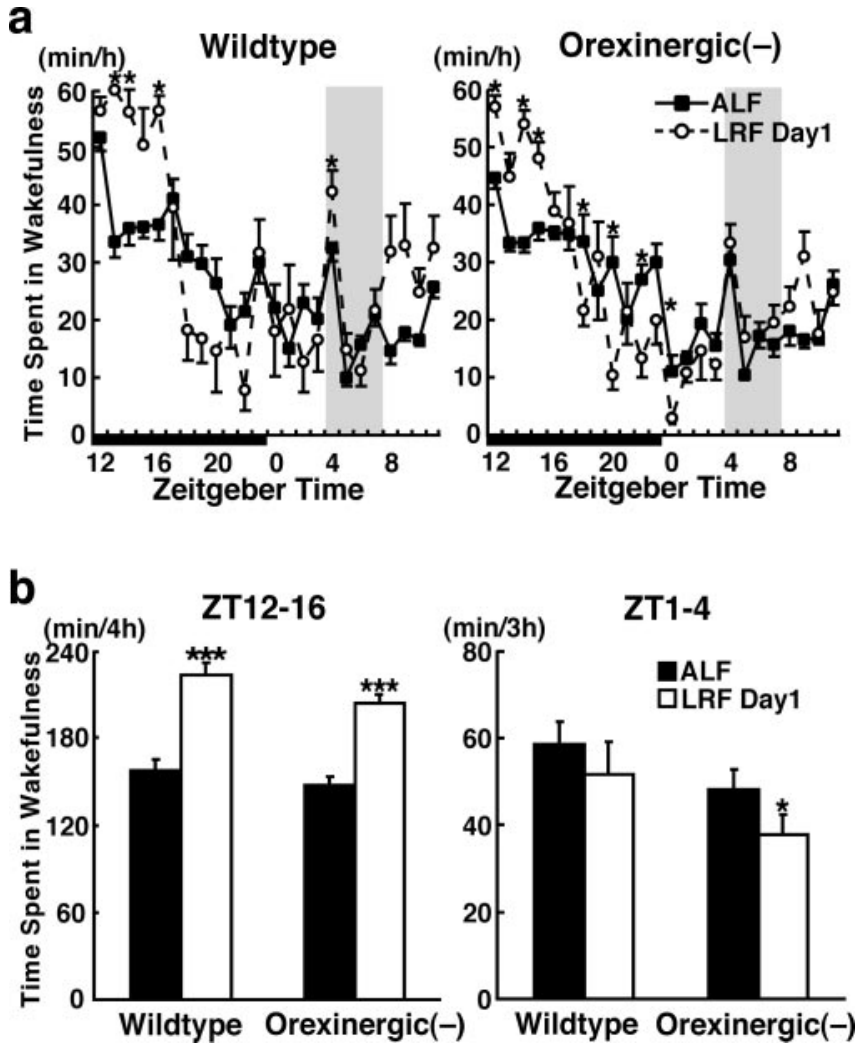

Figure 6. Similar responses to the absence of food by wild-type and orexinergic(-) mice in the first dark phase under light phase-restricted feeding. $a$, Hourly plots of time spent in wakefulness under ALF or on the first day under LRF (see Results and Materials and Methods). The period of food availability is shaded, and the dark phase is indicated by a solid horizontal bar. ${ }^{*} p<0.05$ ALF versus LRF day1; within each genotype; one-way repeated-measures ANOVA (including data for "LRF" and "RLF-fast") and Tukey post hoc tests. $b$, Time spent in wakefulness during the onset of the dark phase (ZT12-16) or the periods of anticipation (ZT1-4) on LRF day 1. ${ }^{*} p<0.05 ;{ }^{* * *} p<0.001$ ALF versus LRF day 1 within each genotype; two-way repeatedmeasures ANOVA. There was no significant interaction between feeding condition and genotype. Values are means $\pm \mathrm{SE}$ ( $n=8$ for each genotype).

ability (Fig. 7a,b). Hence, orexin neurons are essential for exhibiting the normal anticipatory increase in wakefulness regardless of circadian phase. However, orexinergic(-) and wild-type mice showed similar increased wakefulness when no food was given during the expected feeding period (Fig. $7 a, b$, DRF-fast). This was in notable contrast to the response of orexinergic(-) mice to the same situation under LRF, when they exhibited a severely blunted wakefulness response compared with wild-type mice (Fig. 4a, b, LRF-fast). Orexinergic(-) mice maintained a similar $24 \mathrm{hr}$ wakefulness time under DRF compared with that recorded under ALF, whereas wild-type mice demonstrated significantly increased $24 \mathrm{hr}$ wakefulness time because of the additional wakefulness during the period of food anticipation (Fig. 7c). Under DRF, therefore, orexin neurons may enable mice to decrease the homeostatic sleep drive with an increased $24 \mathrm{hr}$ wakefulness time. This is consistent with the hypothesis that orexin neurons are involved in $24 \mathrm{hr}$ sleep homeostasis by having a different influence on wakefulness during the light and dark phases. This follows from the fact that under LRF, in which the $24 \mathrm{hr}$ vigilance state rhythm was reversed in both genotypes, orexinergic (-) mice exhibited less $24 \mathrm{hr}$ wakefulness time, whereas wild-type mice maintained similar $24 \mathrm{hr}$ wakefulness time to that recorded under ALF (Fig. 2a). 
a
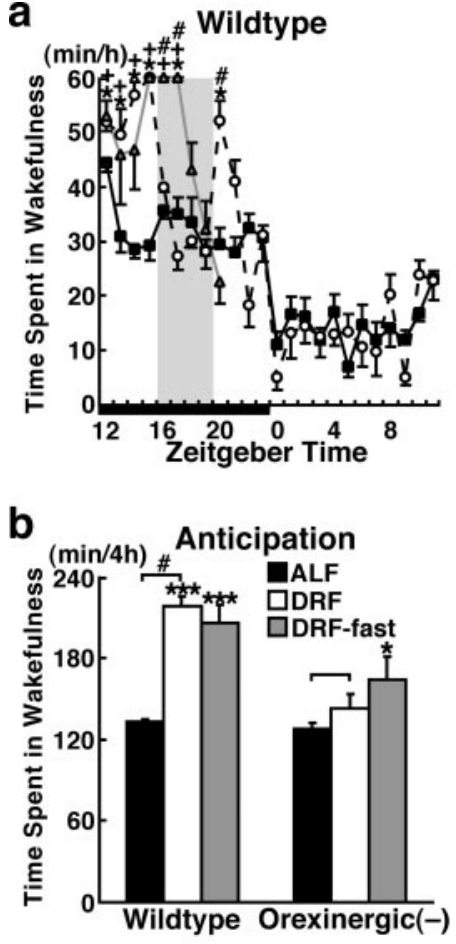

C

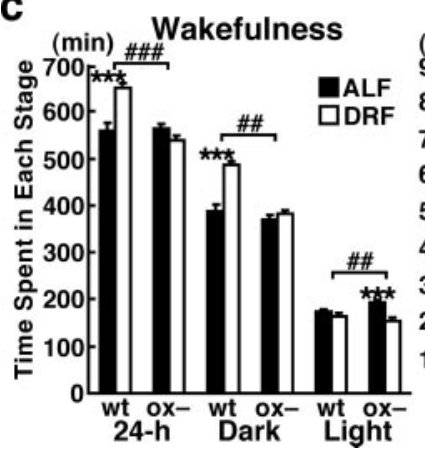

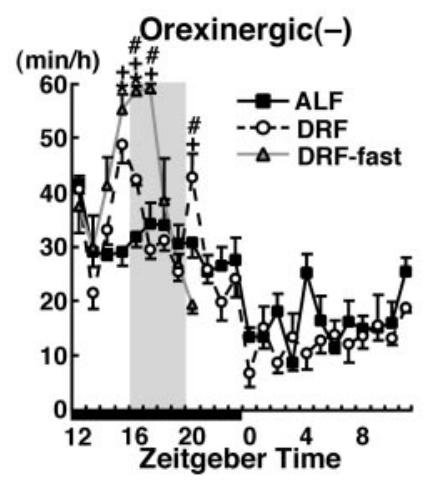

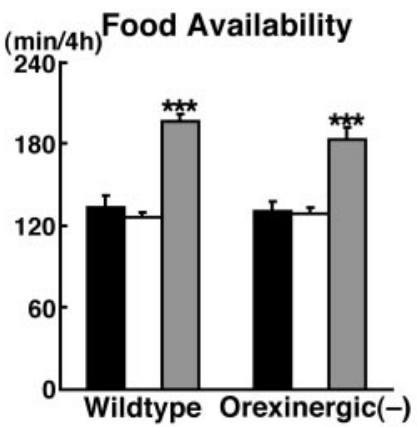

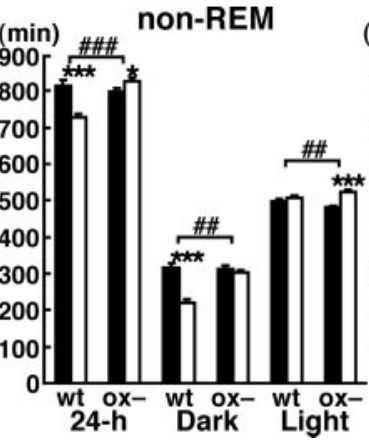

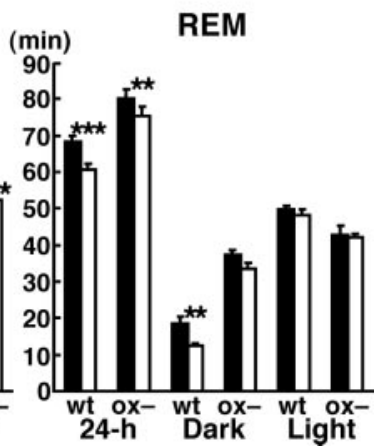

Figure 7. Attenuation of food-anticipatory increase in wakefulness in orexinergic(-) mice under dark phase-restricted feeding. a, Hourly plots of time spent in wakefulness under ALF, DRF, or DRF without food. Under "DRF-fast" at approximately ZT22, mice of both genotypes started to demonstrate uncharacteristic EEG patterns that could not be assigned to any vigilant state. The period of food availability is shaded, and the dark phase is indicated by a solid horizontal bar. ${ }^{*} p<0.05$ ALF versus DRF; $+p<0.05$ ALF versus DRF-fast; \# $<<0.05$ DRF versus DRF-fast; within each genotype; one-way repeated-measures ANOVA and Tukey post hoc tests. $b$, Time spent in wakefulness during the periods of anticipation (ZT12-16) or food availability (ZT16-20). c, Time spent in each stage during $24 \mathrm{hr}$ day ( $24 \mathrm{hr}$ ), $12 \mathrm{hr}$ dark phase (Dark), and $12 \mathrm{hr}$ light phase (Light). Under DRF, wild-type mice (wt) but not orexinergic(-) mice (ox-) demonstrated increased $24 \mathrm{hr}$ wakefulness time compared with ALF. In $b$ and $c,{ }^{*} p<0.05 ;{ }^{* *} p<0.01$ ${ }^{* * *} p<0.001$ ALF versus DRF within each genotype; ${ }^{* \#} p<0.01 ;{ }^{* \# \#} p<0.001$ interaction of feeding condition and genotype; two-way repeated-measures ANOVA. Values are means \pm SE ( $n=6$ for wild-type mice; $n=5$ for orexinergic(-) mice).

suggest that orexin neurons are not required for the entrainment of the putative FEO by feeding, but essential for the execution of signals from the FEO to increase wakefulness and activity during the foodanticipatory period. Indeed, orexin neurons are neuroanatomically well placed to modulate the sleep-regulating monoaminergic and cholinergic systems (Chemelli et al., 1999; Saper et al., 2001; Mieda and Yanagisawa, 2002) in accordance with putative signals from the FEO. Deficits in food-anticipatory behavior in orexinergic(-) mice appear to be mechanistically different from those seen in mice deficient for NPAS2, a paralog of Clock, in which the defect lies in the NPAS2-driven molecular clock per se, a clock that is apparently essential for adaptation to restricted feeding (Dudley et al., 2003). It is also important to note that the circadian modulation of behaviors driven by the LEO is essentially independent of orexin neurons. Thus, mice are more active and wakeful during the dark phase than during the light phase with or without orexin neurons (Figs. 2-4) (Hara et al., 2001). Moreover, circadian modulation of locomotor activities under dark:dark free-running conditions was essentially unaltered in orexin knockout mice (M. Mieda and M. Yanagisawa, unpublished data). Thus, orexin neurons are essential for the execution of FEOdriven but not LEO-driven behaviors.

Orexinergic(-) mice have a normal lifespan and showed normal changes in food intake and body weight even though they lacked most of the food-anticipatory increase in locomotor activity and wakefulness under restricted feeding. These findings raise the question as to the adaptive value of FAA. Under the conditions of restricted feeding in this study, food was given to mice at precisely the same time every day in their home cages. Therefore, food intake of these mice deviated little from normal during the period of food availability. However, in the wild, even if food availability was restricted to a certain

\section{Discussion}

In this study, we used orexinergic(-) transgenic mice, in which orexin neurons specifically and postnatally degenerate (Hara et al., 2001), to elucidate the role of orexin neurons in the circadian adaptation to restricted feeding, particularly with respect to vigilance state regulation. Changes caused by LRF in food consumption, as well as in the circadian expression of the $m P e r 2$ gene in the liver and various brain regions, were indistinguishable between wild-type and orexinergic(-) mice. The circadian sleep-wake pattern was also similarly reversed under LRF in both genotypes. In contrast, the food-anticipatory increase in locomotor activity and wakefulness under LRF was severely impaired in orexinergic (-) mice. Fos-IR in orexin neurons markedly increased during the food-anticipatory period in wild-type mice. These findings period of the day, it could still be uncertain and deviate temporally and spatially. Thus, increasing activity and wakefulness in the hours immediately preceding expected food availability would aid survival by foraging for food within a variable window of opportunity.

\section{Orexin neurons in an efferent pathway of the putative FEO}

We have shown previously that orexin neurons monitor peripheral indicators of nutritional state and mediate an adaptive augmentation of wakefulness in response to negative energy balance (Yamanaka et al., 2003). Thus, the possibility arises that orexin neurons are sensing the depletion of nutrients and thus increasing wakefulness during the hours that precede food availability under restricted feeding, rather than conveying signals from a 
bona fide circadian oscillator. However, we consider that this possibility is unlikely because mice exhibited no appreciable increase in wakefulness immediately before food availability on the first day of restricted feeding, when the food-entrained behavioral rhythm had not been established (Fig. 6b, ZT1-4). Furthermore, mice gradually decreased wakefulness when food was not available during the expected feeding period, a situation that must further deplete nutrients. Previous studies have also demonstrated that FAA is not simply triggered each day when energy depletion reaches some threshold before food availability (Mistlberger, 1994).

Orexinergic(-) mice are known to be hypometabolic, at least on one genetic background (Hara et al., 2001). Thus, it is possible that the hypometabolism of orexinergic $(-)$ mice simply reduces the need for, and anticipation of, food. We consider that this explanation is also unlikely. First, changes in food intake and body weight under LRF were comparable between wild-type and orexinergic(-) mice, suggesting that they responded similarly to LRF with respect to overall energy balance. Second, orexinergic(-) mice responded to certain behavioral contexts, such as actual food availability, by increasing wakefulness to an extent comparable to that observed in wild-type mice (Fig. $4 b$ ). This suggests that the desire for food is similar in the two genotypes.

The TMN is a likely downstream target of orexin neurons for the elicitation of arousal during food anticipation, because (1) TMN histaminergic neurons have been reported to be critical for the maintenance of wakefulness (Parmentier et al., 2002); (2) they receive a relatively dense projection of orexin neurons (Peyron et al., 1998; Chemelli et al., 1999; Date et al., 1999); (3) they express $\mathrm{OX}_{2} \mathrm{R}$ receptors (Marcus et al., 2001; Willie et al., 2003); and (4) they at least in part mediate the arousing effects of centrally administered orexin-A (Huang et al., 2001; Yamanaka et al., 2002). The increase of Fos-IR cell number in this area suggested that TMN neurons are indeed activated during the foodanticipatory period under LRF. Nevertheless, we failed to detect any difference in the activation of TMN neurons between wildtype and orexinergic (-) mice, despite the marked attenuation of food-anticipatory wakefulness in orexinergic (-) mice. This observation suggests that orexin neurons are not required for the activation of TMN neurons during food anticipation and that activation of TMN neurons is not sufficient for exhibiting normal food-anticipatory activity and wakefulness. Thus, contrary to expectation, TMN neurons are unlikely to be a principal site for mediating the signal from orexin neurons in eliciting FAA. During a preliminary scan of sections covering the entire brain for Fos-IR, we did not find any areas with marked differences between genotypes in Fos induction during food anticipation. Additional detailed studies are therefore necessary to identify the neural circuits involved in the regulation of food anticipation by orexin neurons.

\section{Modulation of orexin neuron-mediated vigilance regulation by circadian phase}

Comparisons between the adaptation of orexinergic(-) mice to LRF and DRF suggested that the contribution of orexin neurons to increasing and maintaining wakefulness is modified by circadian phase. The reduced total $24 \mathrm{hr}$ wakefulness time under LRF in orexinergic (-) mice is particularly interesting because $24 \mathrm{hr}$ sleep homeostasis has been thought to be unaltered in murine, canine, and human narcoleptics, because their daily wakefulness times are comparable to normal individuals under baseline conditions (Hishikawa et al., 1976; Mitler and Dement, 1977; Montplaisir et al., 1978; Chemelli et al., 1999; Hara et al., 2001; Willie et al., 2003). Equally interesting is the finding that the $24 \mathrm{hr}$ wakefulness time was significantly increased under DRF in wild-type mice. Although the mechanism remains undefined, this shift in the set point for $24 \mathrm{hr}$ wakefulness time apparently requires the presence of orexin neurons. Because we performed all experiments under $12 \mathrm{hr}$ light/dark conditions in the present study, we were not able to discriminate between the input from the central clock in the SCN and the presence of light, as the relevant modulator. Circadian signals from the SCN are known to suppress locomotor activity (Buijs and Kalsbeek, 2001) and wakefulness (Mistlberger et al., 1983; Tobler et al., 1983; Dijk and Czeisler, 1995) during the rest phase. Independently of the SCN, however, light also has a direct suppressive effect on locomotor activity (Redlin, 2001) and probably on wakefulness (Fishman and Roffwarg, 1972; Benca et al., 1998) in nocturnal animals (i.e., the masking effect).

Whereas orexin neurons were shown in this study to be essential for the normal food-anticipatory increase in wakefulness regardless of the light/dark phase, the absence of orexin neurons made no appreciable difference to the increase in wakefulness during actual food availability under restricted feeding in the light or dark phase. During food availability, physical movement associated with the ingestion of food (Matsumoto et al., 2002), as well as the odor and/or sight of food, might help the mice to maintain arousal via an orexin-independent mechanism. Thus, the contribution of orexin neurons to the promotion of wakefulness seems to be context dependent: it may vary according to the overall activity of other neuronal populations that regulate the sleep-wake cycle.

\section{References}

Benca RM, Gilliland MA, Obermeyer WH (1998) Effects of lighting conditions on sleep and wakefulness in albino Lewis and pigmented Brown Norway rats. Sleep 21:451-460.

Beuckmann CT, Sinton CM, Williams SC, Richardson JA, Hammer RE, Sakurai T, Yanagisawa M (2004) Expression of a poly-glutamineataxin-3 transgene in orexin neurons induces narcolepsy-cataplexy in the rat. J Neurosci 24:4469-4477.

Buijs RM, Kalsbeek A (2001) Hypothalamic integration of central and peripheral clocks. Nat Rev Neurosci 2:521-526.

Chemelli RM, Willie JT, Sinton CM, Elmquist JK, Scammell T, Lee C, Richardson JA, Williams SC, Xiong Y, Kisanuki Y, Fitch TE, Nakazato M, Hammer RE, Saper CB, Yanagisawa M (1999) Narcolepsy in orexin knockout mice: molecular genetics of sleep regulation. Cell 98:437-451.

Cirelli C, Tononi G (2000) On the functional significance of c-fos induction during the sleep-waking cycle. Sleep 23:453-469.

Damiola F, Le Minh N, Preitner N, Kornmann B, Fleury-Olela F, Schibler U (2000) Restricted feeding uncouples circadian oscillators in peripheral tissues from the central pacemaker in the suprachiasmatic nucleus. Genes Dev 14:2950-2961.

Date Y, Ueta Y, Yamashita H, Yamaguchi H, Matsukura S, Kangawa K, Sakurai T, Yanagisawa M, Nakazato M (1999) Orexins, orexigenic hypothalamic peptides, interact with autonomic, neuroendocrine and neuroregulatory systems. Proc Natl Acad Sci USA 96:748-753.

de Lecea L, Kilduff TS, Peyron C, Gao X, Foye PE, Danielson PE, Fukuhara C, Battenberg EL, Gautvik VT, Bartlett FS, 2nd, Frankel WN, van den Pol AN, Bloom FE, Gautvik KM, Sutcliffe JG (1998) The hypocretins: hypothalamus-specific peptides with neuroexcitatory activity. Proc Natl Acad Sci USA 95:322-327.

Dijk DJ, Czeisler CA (1995) Contribution of the circadian pacemaker and the sleep homeostat to sleep propensity, sleep structure, electroencephalographic slow waves, and sleep spindle activity in humans. J Neurosci 15:3526-3538.

Dudley CA, Erbel-Sieler C, Estill SJ, Reick M, Franken P, Pitts S, McKnight SL (2003) Altered patterns of sleep and behavioral adaptability in NPAS2deficient mice. Science 301:379-383.

Fishman R, Roffwarg HP (1972) REM sleep inhibition by light in the albino rat. Exp Neurol 36:166-178. 
Franklin KJ, Paxinos G (2001) The mouse brain. San Diego: Academic.

Hagan JJ, Leslie RA, Patel S, Evans ML, Wattam TA, Holmes S, Benham CD, Taylor SG, Routledge C, Hemmati P, Munton RP, Ashmeade TE, Shah AS, Hatcher JP, Hatcher PD, Jones DN, Smith MI, Piper DC, Hunter AJ, Porter RA, Upton N (1999) Orexin A activates locus coeruleus cell firing and increases arousal in the rat. Proc Natl Acad Sci USA 96:10911-10916.

Hara J, Beuckmann CT, Nambu T, Willie JT, Chemelli RM, Sinton CM, Sugiyama F, Yagami K, Goto K, Yanagisawa M, Sakurai T (2001) Genetic ablation of orexin neurons in mice results in narcolepsy, hypophagia, and obesity. Neuron 30:345-354.

Hishikawa Y, Wakamatsu H, Furuya E, Sugita Y, Masaoka S (1976) Sleep satiation in narcoleptic patients. Electroencephalogr Clin Neurophysiol 41:1-18.

Huang ZL, Qu WM, Li WD, Mochizuki T, Eguchi N, Watanabe T, Urade Y, Hayaishi O (2001) Arousal effect of orexin A depends on activation of the histaminergic system. Proc Natl Acad Sci USA 98:9965-9970.

Inzunza O, Seron-Ferre MJ, Bravo H, Torrealba F (2000) Tuberomammillary nucleus activation anticipates feeding under a restricted schedule in rats. Neurosci Lett 293:139-142.

Lin L, Faraco J, Li R, Kadotani H, Rogers W, Lin X, Qiu X, de Jong PJ, Nishino S, Mignot E (1999) The sleep disorder canine narcolepsy is caused by a mutation in the hypocretin (orexin) receptor 2 gene. Cell 98:365-376.

Marcus JN, Aschkenasi CJ, Lee CE, Chemelli RM, Saper CB, Yanagisawa M, Elmquist JK (2001) Differential expression of orexin receptors 1 and 2 in the rat brain. J Comp Neurol 435:6-25.

Matsumoto Y, Mishima K, Satoh K, Shimizu T, Hishikawa Y (2002) Physi$\mathrm{cal}$ activity increases the dissociation between subjective sleepiness and objective performance levels during extended wakefulness in human. Neurosci Lett 326:133-136.

Mieda M, Yanagisawa M (2002) Sleep, feeding, and neuropeptides: roles of orexins and orexin receptors. Curr Opin Neurobiol 12:339-345.

Mignot E, Taheri S, Nishino S (2002) Sleeping with the hypothalamus: emerging therapeutic targets for sleep disorders. Nat Neurosci [Suppl] 5:1071-1075.

Mistlberger RE (1994) Circadian food-anticipatory activity: formal models and physiological mechanisms. Neurosci Biobehav Rev 18:171-195.

Mistlberger RE, Bergmann BM, Waldenar W, Rechtschaffen A (1983) Recovery sleep following sleep deprivation in intact and suprachiasmatic nuclei-lesioned rats. Sleep 6:217-233.

Mistlberger RE, Antle MC, Kilduff TS, Jones M (2003) Food- and lightentrained circadian rhythms in rats with hypocretin-2-saporin ablations of the lateral hypothalamus. Brain Res 980:161-168.

Mitler MM, Dement WC (1977) Sleep studies on canine narcolepsy: pattern and cycle comparisons between affected and normal dogs. Electroencephalogr Clin Neurophysiol 43:691-699.

Montplaisir J, Billiard M, Takahashi S, Bell IR, Guilleminault C, Dement WC (1978) Twenty-four-hour recording in REM-narcoleptics with special reference to nocturnal sleep disruption. Biol Psychiatry 13:73-89.

Parmentier R, Ohtsu H, Djebbara-Hannas Z, Valatx JL, Watanabe T, Lin JS (2002) Anatomical, physiological, and pharmacological characteristics of histidine decarboxylase knock-out mice: evidence for the role of brain histamine in behavioral and sleep-wake control. J Neurosci 22:7695-7711.

Peyron C, Tighe DK, van den Pol AN, de Lecea L, Heller HC, Sutcliffe JG, Kilduff TS (1998) Neurons containing hypocretin (orexin) project to multiple neuronal systems. J Neurosci 18:9996-10015.

Redlin U (2001) Neural basis and biological function of masking by light in mammals: suppression of melatonin and locomotor activity. Chronobiol Int 18:737-758.

Reick M, Garcia JA, Dudley C, McKnight SL (2001) NPAS2: an analog of clock operative in the mammalian forebrain. Science 293:506-509.

Reppert SM, Weaver DR (2002) Coordination of circadian timing in mammals. Nature 418:935-941.

Sakurai T, Amemiya A, Ishii M, Matsuzaki I, Chemelli RM, Tanaka H, Williams SC, Richardson JA, Kozlowski GP, Wilson S, Arch JR, Buckingham RE, Haynes AC, Carr SA, Annan RS, McNulty DE, Liu WS, Terrett JA, Elshourbagy NA, Bergsma DJ, Yanagisawa M (1998) Orexins and orexin receptors: a family of hypothalamic neuropeptides and G proteincoupled receptors that regulate feeding behavior. Cell 92:573-585.

Saper CB, Chou TC, Scammell TE (2001) The sleep switch: hypothalamic control of sleep and wakefulness. Trends Neurosci 24:726-731.

Scammell TE (2003) The neurobiology, diagnosis, and treatment of narcolepsy. Ann Neurol 53:154-166.

Stephan FK (2002) The "other" circadian system: food as a Zeitgeber. J Biol Rhythms 17:284-292.

Stokkan KA, Yamazaki S, Tei H, Sakaki Y, Menaker M (2001) Entrainment of the circadian clock in the liver by feeding. Science 291:490-493.

Tobler I, Borbely AA, Groos G (1983) The effect of sleep deprivation on sleep in rats with suprachiasmatic lesions. Neurosci Lett 42:49-54.

Wakamatsu H, Yoshinobu Y, Aida R, Moriya T, Akiyama M, Shibata S (2001) Restricted-feeding-induced anticipatory activity rhythm is associated with a phase-shift of the expression of mPerl and mPer2 mRNA in the cerebral cortex and hippocampus but not in the suprachiasmatic nucleus of mice. Eur J Neurosci 13:1190-1196.

Willie JT, Chemelli RM, Sinton CM, Tokita S, Williams SC, Kisanuki YY, Marcus JN, Lee C, Elmquist JK, Kohlmeier KA, Leonard CS, Richardson JA, Hammer RE, Yanagisawa M (2003) Distinct narcolepsy syndromes in Orexin receptor-2 and Orexin null mice: molecular genetic dissection of Non-REM and REM sleep regulatory processes. Neuron 38:715-730.

Yamanaka A, Tsujino N, Funahashi H, Honda K, Guan JL, Wang QP, Tominaga M, Goto K, Shioda S, Sakurai T (2002) Orexins activate histaminergic neurons via the orexin 2 receptor. Biochem Biophys Res Commun 290:1237-1245.

Yamanaka A, Beuckmann CT, Willie JT, Hara J, Tsujino N, Mieda M, Tominaga M, Yagami K, Sugiyama F, Goto K, Yanagisawa M, Sakurai T (2003) Hypothalamic orexin neurons regulate arousal according to energy balance in mice. Neuron 38:701-713.

Zylka MJ, Shearman LP, Weaver DR, Reppert SM (1998) Three period homologs in mammals: differential light responses in the suprachiasmatic circadian clock and oscillating transcripts outside of brain. Neuron 20: 1103-1110. 